\title{
Acute Effects of Alcohol on Measures of Walking Performance
}

\author{
R. A. M. Gregson, D. A. R. Smith, E. R. Strelow, and J. A. Brabyn \\ University of Canterbury, New Zealand
}

An on-line system for continuously monitoring 10 different quantitative measures of walking movements to a high fidelity was employed in a study comparing the effects of tolerance for alcohol and dosage level in $\mathrm{mg} / \mathrm{kg}$ body weight over an $80 \mathrm{~min}$ ute period following drinking. The data were subjected to multivariate analyses of covariance. Complex changes in walking were detected-some transient-during the period of observation. Different measures were optimally sensitive in monitoring a straight walking task and a blindfolded complex walk. The consequent problems of validation and generalization are noted.

It has been known for centuries that the ingestion of sufficient quantities of alcohol will impair the gait and the accuracy of motor selfregulation in walking movements. The very detailed monitoring of the action of alcohol on these walking skills and the quantification of individual differences in the dynamic execution of walking movements has, however, awaited the development of modern instrumentation and on-line computer analysis.

An open space facility, originally developed to study locomotion in the blind and its facilitation with electronic sensors (Kay, 1974) and described in detail by Strelow, Brabyn, \& Clark (1976), has made it possible to accurately locate

APPLIED PSYCHOLOGICAL MEASUREMENT

Vol. 2, No. 2 Spring 1978 pp. 203-219

(C) Copyright 1978 West Publishing Co. and monitor the head location of an erect walking or standing subject over an area of 80 square meters, with a position error of less than $2.5 \mathrm{~cm}$. The signals from subjects who can walk normally and unconstrainedly provide a continuous record of their coordinates in the area of the laboratory floor about $8 \mathrm{~m} \times 9 \mathrm{~m}$. The system is capable of tracking a subject who is accelerating at not more than $3 \mathrm{~m} / \mathrm{sec}^{2}$ in any direction in the horizontal plane. The signals are fed into an analog-digital computer, so that path and velocity components of performance can be analyzed mathematically. An optimal path can be specified in advance; measures can be made of departures from the path and quantitative assessment of smoothness in motion, speed variations, and other parameters independent of the actual spatial path followed by the subject.

All such measures can be regarded as integrals over some measure of performance efficiency, but the choice of appropriate functions for measuring performance degradation under the acute influence of alcohol is not settled. Consequently, this study aims, in part, to provide a relative evaluation of some possible quantitative measures.

This investigation was set up to examine the form and extent of individual differences in the deterioration (and recovery) of walking performance during the first 80 minutes after ingestion, as a function of both dosage and estimated toler- 
ance. In practically all aspects, there were no quantitative precedents to guide the investigators concerning the expected magnitude or dynamic form of the performance impairments, if any. While tests of intoxication and subsequent motor incapacity have been in use by medical workers and police or traffic officers for some years, none of these tests are quantified in a manner relevant to our terms of reference. Analogous results in the effects of alcohol on tracking tasks (Reid \& Ibrahim, 1975) are indicative of the dynamic complexity of the problem. They are not definitive here, however, because walking in a straight line and tracking manual controls in car steering are not dynamically equivalent (Sheridan \& Ferrell, 1974). Also, the large individual differences which are expected and the rapid changes which are possible during the first 80 minutes after ingestion impose constraints on experimental design and data analysis.

\section{Method}

\section{Apparatus}

Testing for the effects of alcohol by the use of sensorimotor tasks has been widespread, but has received comparatively little study. The main reason for this has been the absence of a measurement device that can measure with accuracy and, in turn, calculate higher order measures. Previous researchers have used wooden beams with an off/on criterion (Fregly, Bergsteadt, \& Graybiel, 1967), various balance platforms using the angle of the plane as a measurement (Begbie, 1966), or a variation of a complex coordination task (Powell, Goodwin, \& Bremer, 1973; Vogel, 1958). Two researchers (Miles, 1950; Schmidt, 1956) used a similar mechanism to that used in this study-a mechanical system of wires attached to the head of the subject and linked to recording facilities. The present facility represents a considerable advance in accuracy and sophistication.

The apparatus here, described by Strelow et al. (1976), uses electric-mechanical transducers linked with an EAI 640 Digital Computer to determine position in an eight-meter-square laboratory five times a second. The computer then analyzes the completed run in terms of 10 measures:

1. Average speed, which is non-directional.

2. Acceleration, average magnitude.

3. Standard deviation of speed.

4. Average deviation about average speed.

5. Root mean square deviation from a straight line between two arbitrary, but specified, reference points in the field of locomotion.

6. Average deviation about the straight line defined in 5 .

7. Root mean square deviation from a straight line fitted through the plotted path.

8. Average deviation about 7 .

9. Average curvature between each pair of plotted points in radians per second.

10. Surplus turning, a measure which relates to excessive rotatory movement.

\section{Experimental Procedure}

Subjects were contacted at least a day prior to the experimental session, and a check was made on whether or not each subject was on any medication; none were. Subjects were instructed to have no alcohol on the day of the experiment, to take lunch between 12:30 and 1 P.M., and to have no food or coffee following this. The subjects were run two at a time.

On arrival at the laboratory at 4 P.M., each pair of subjects was instructed and given practice on three sensory-motor tasks:

1. A straight line walk in a relaxed manner from one marker pole to another, the distance apart being about $10 \mathrm{~m}$., taking one step per metronome beat $(1.5 \mathrm{~Hz})$.

2. The Romberg test, in which the subject stood in one spot in the middle of the room. blindfolded, with arms crossed. During a countdown from " 5 " to " 0 " the subject would place one foot (whichever was pre- 
ferred) in front of the other and from " 0 " would be monitored for sway for 30 seconds. The subject was instructed to remain as still as possible.

3. A "complex walk" from one pole to another with arms crossed, walking heel to toe in time to the metronome $(1.5 \mathrm{~Hz})$. Subjects were blindfolded in this task, also.

For both Tasks 1 and 3 a countdown was given by the experimenter, whereas the countdown for Task 2 was given by the computer operator using an intercom.

The subjects were first tested sober and then given either a "dose" of $1 \mathrm{gm} / \mathrm{kg}$ body weight of pure alcohol (vodka, orange juice, and ginger ale to $500 \mathrm{ml}$ ) or a "placebo" of $0.1 \mathrm{gm} / \mathrm{kg}$ body weight of pure alcohol (vodka, orange juice, and ginger ale to $500 \mathrm{ml}$ ) and asked to drink it all within 10 minutes, which all except two subjects managed to do. The dose of alcohol was allocated by random procedures and made up by an independent person in order to make the experiment blind. The subjects knew only that the experiment included the testing of the effects of alcohol over time.

The subjects were next separated from each other and tested every ten minutes on the three sensorimotor tasks, which were done in a balanced randomly allocated design so that order effects were partially equated. It was suspected during pilot studies that the atmosphere of the experiment and attitude of the experimenter would influence the performance, so an attitude which, although interested, was as clinically detached as possible was taken by the experimenter. After being tested for eighty minutes (intoxicated) the subjects were informed that the experiment was finished. They were shown, in part, the results of the experiment to date and their own results as far as possible. Finally, they were told how much alcohol they had consumed and the general purpose of the experiment. Since some subjects were over the legal blood alcohol limit for driving, they were informed of their estimated blood alcohol level, using the ap- proximations of Watson and Batt (1976), and were paid $\$ 2$ in expenses to take alternative transportation home. The two low tolerance subjects were taken home.

\section{Subjects}

A group of 104 students from either psychology or engineering were first surveyed by questionnaire to determine (1) the self-perceived drinking behavior of students and (2) those who were willing to partake in an experiment "involving them drinking a quantity of alcohol and being tested." Twelve students who reported the heaviest consumption and 12 who reported the lightest were chosen as the high and low tolerance subjects, respectively.

Analysis of the self-reported drinking was made on the basis of both its frequency and quantity, following Bowman, Stein, and Newton, (1975). Powell et al. (1973) have stated that experience with alcohol leads to tolerance. For the purpose of this experiment, which used the extremes of the surveyed sample in relation to alcohol intake, the test is adequate. For any more definitive test of tolerance, a more complete drinking history would need to be considered and a wider age range of subjects used.

Population characteristics. The measure of drinking behavior, an estimate of mean interdrinking interval, biased the estimates of drinking frequency upwards. Graphed on a log scale of frequency, however, it facilitated the identification of the heavier drinker, as well as the group of very light drinkers or abstainers ( 9 persons). Thus, for the restricted purpose of selecting subjects, the questionnaire was adequate; the distribution of reported drinking behavior is shown in Figure 1.

Subject characteristics. The high tolerance subjects ranged from one subject who drank regularly and reasonably heavily $(74 \mathrm{gm} /$ day) to one who drank regularly, but only twice a week, and then only $35 \mathrm{gm} / \mathrm{occasion}$. By contrast, the low tolerance drinkers ranged from one subject who drank fortnightly, but very little, to a sub- 


\section{Figure 1}

Frequency Distribution of the Most Recent Self-reported Interval since Last Consumption of Alcoholic Drink:

Most Recent Reports are Subdivided by Quantity Consumed
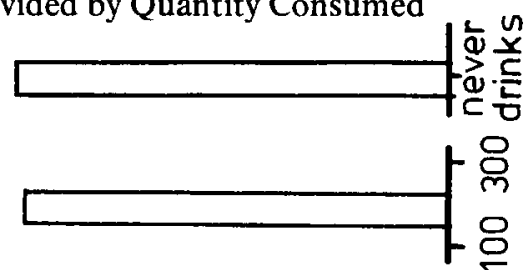

.

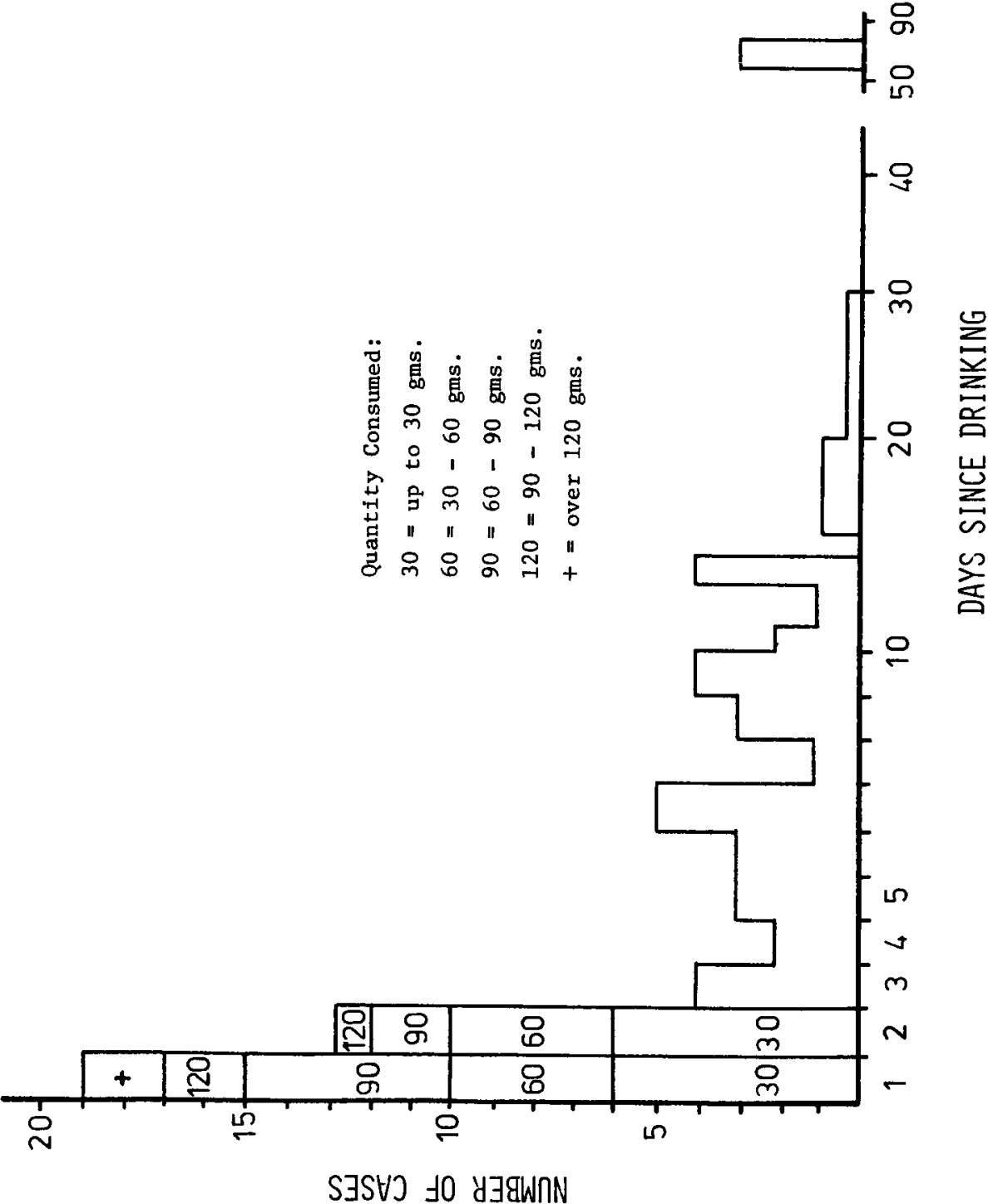


ject who had drunk only once, and then extremely little. The questionnaire on self-assessed consumption was administered twice: first upon selection and second upon testing the selected subjects. The average of the two self-reports was taken as the consumption estimate; for low drinkers the two reports agreed completely. The two groups were, therefore, completely different in their self-reported exposure to alcohol.

\section{Tasks Used}

Comparison was made of the effects of dose levels of $1 \mathrm{gm} / \mathrm{kg}$ and $0.1 \mathrm{gm} / \mathrm{kg}$ body weight tracked over time on the low and high tolerance groups of subjects, with five subjects in each dose $x$ tolerance condition. The sensorimotor tasks which were chosen, subsequent to a pilot study of eight subjects who were not used in the main study, have been given above. The straight line walk is a well-practiced common task and yet little studied (Fregly et al., 1967). The Romberg test was included because of its previous use (Carlson et al., 1934, p. 85; Goldberg, 1943; Laves, 1955; Schmidt, 1956; Pihkamen, 1957b; Hebbelinck, 1963; Begbie, 1966; Idestom \& Cadenium, 1968) and its medical use as an index of ataxia. The complex walk was postulated by the first author to demand effort from the subject while sober, and thus be possibly less susceptible to changes in the subject's concentration and effort during the experiment. It was novel and has not previously been used in alcohol research; it, however, could be considered a mobile version of the Romberg test with auditory perceptual load added, because the subjects walk blindfolded towards the sound source provided by the ticking of a metronome.

\section{Data Analysis}

Powell et al., (1972) found that experienced drinkers tested after taking alcohol had better simple motor performance and engaged in less risk-taking behavior than relatively inexperienced drinkers. It may, therefore, be predicted that at least on some performance indices, experienced drinkers would modify their movements in such a way that disclosed less loss of precise movement control than would be shown in inexperienced drinkers. It is necessary to examine each task and measure with this general prediction in mind.

The output data for each subject on each task. on each measure, at serial points in time, were used as input to a series of multivariate analyses of covariance (MANOCOVAs). These were interpreted in conjunction with the graphs of mean effects (contrasts). A series of MANOCOVAs were conducted for both the straight walk and complex walk tasks; these will be described separately. The Romberg task is not examined in this report.

\section{Results}

\section{Straight Line Walking}

The data were pooled over subjects, giving five entries per cell in a design of

1. Two levels of tolerance for alcohol (coded as Factor C);

2. Two levels of dosage (coded as Factor D); and

3. Time elapsed after drinking, 9 points at $\mathbf{1 0}$ minute intervals (Coded as Factor T);

with 10 variables (the measures specified, which will be referred to by abbreviated names consistent with their definitions above). The analysis was treated as having multivariate components $\mathrm{C}, \mathrm{D}, \mathrm{CD}$, and $\mathrm{T}$ within $\mathrm{CD}$.

MANOVA 1 , with no adjustment for covariates. Overall effects for this analysis were assessed by Wilks' Lambda and expressed as canonical correlations. Table 1 gives a summary of the results. The major effects, CD, D, and C are important, although there was no average change over time, $T^{1}$. However, within these ef-

'Factor $T$ is omitted from Tables 1 and 2 since no significant changes in it were found in these analyses. 
Table 1

Results of the Multivariate Analysis of Variance Tests of Effects for the Straight Walk, Canonical Correlations $\left(R_{c}\right)$ and Correlations of Variables with Canonical Variates ${ }^{c}\left(\mathrm{r}_{\mathrm{xv}}\right)$

\begin{tabular}{|c|c|c|c|}
\hline Effect & F-Ratio & $p$ & $\mathrm{R}_{\mathrm{C}}$ \\
\hline \multicolumn{4}{|l|}{ General Effects } \\
\hline Source & $F(10,135)$ & & \\
\hline CD & 4.32 & .001 & .493 \\
\hline $\mathrm{D}$ & 22.08 & .001 & .788 \\
\hline $\mathrm{C}$ & 5.41 & .001 & .535 \\
\hline $\begin{array}{c}\text { Single Variable Effects } \\
\text { Within CD (tolerance- } \\
\text { dosage interaction) }\end{array}$ & $F(1,144)$ & & $r_{x V}$ \\
\hline 2. Accel. Av. Mag. & 1.17 & .190 & .362 \\
\hline 4. Av. Dev. about Av. Speed & 25.97 & .001 & .750 \\
\hline 5. R.M.S. Dev. & 4.11 & .045 & .298 \\
\hline 6. Av. Dev. from S. Line & 4.18 & .043 & .001 \\
\hline $\begin{array}{cl}\text { 7. } & \text { R.M.S. Dev. } \\
\text { Within } & \text { D (dosage leve1s) }\end{array}$ & 0.01 & .908 & .159 \\
\hline 1. Av. Speed & 48.83 & .001 & -.455 \\
\hline 2. Acce1. Av. Mag. & 22.65 & .001 & .310 \\
\hline S.D. of speed & 25.93 & .001 & .332 \\
\hline Av. Dev. about Av. Speed & 68.64 & .001 & .540 \\
\hline R.M.S. Dev. & 15.43 & .001 & .256 \\
\hline 7. Av. Dev. from S. Line & 17.35 & .001 & .271 \\
\hline 9. Surp1. Turn. & 20.41 & .001 & .294 \\
\hline Av. Curv. & 23.79 & .001 & .318 \\
\hline Within C (tolerance) & & & \\
\hline 2. Acce1. Av. Mag. & 5.01 & .027 & .295 \\
\hline S.D. of Speed & 9.92 & .002 & .415 \\
\hline Av. Dev. about Av. Speed & 7.15 & .008 & -.352 \\
\hline 7. R.M.S. Dev. & 22.29 & .007 & .622 \\
\hline 8. Av. Dev. from S. Line & 19.29 & .001 & .578 \\
\hline
\end{tabular}

Note. Contrasts not shown were nonsignificant.

fects, strongly dominated by $\mathrm{D}$, the results were very complex because some component measures were important in the context of one independent variable, while others were important in another. All these effects were expressed as averaged over time.

For $\mathrm{CD}$, the tolerance-dose interaction, the dominant measure sensitive to treatment was
Av. Dev. about Av. Speed; this also played a large role in the canonical discriminant variable for CD. For D, 8 of the 10 measures served as discriminators between levels, as against 5 for CD. Here Av. Dev. about Av. Speed again dominated; but Av. Speed, which differed in the opposite direction with increased dosage (more alcohol slowed up walkers and made them wobble 
more), was also important. For $\mathrm{C}$ the situation was simpler with only 5 measures discriminating; the dominant ones were S.D. of Speed, R.M.S. Dev. S.L., and Av. Dev. from St. Line.

It is immediately apparent that average impairment of motor performance was a complex function both of tolerance and of dosage; the dominant measure which accounted for differences in performance with changes in treatment can change, and come or go, with the treatment factors considered.

Of the measures taken, speed was the most basic even though it may not show much change due to the standardization imposed by the metronome; change due to pace length will affect all of the other measures. Thus, in the light of Powell et al.'s (1973) suggestion that experienced drinkers take less risks, it was not surprising to find the speed of the high tolerance subjects steadily dropping. Presumably, this is a learned strategy to compensate for the effect of the alcohol, since no such slowing down was evident in the low tolerance subjects (Figure 2). Consequently, the standard deviation of speed was higher for the dosed low tolerance subjects, but there was no difference between the dosed high tolerance subjects and the placebo groups (Figure 3).

However, as effort was given to maintaining a walking style by high tolerance subjects, their deviation from the desired straight line was considerably higher most of the time than either the placebos or low tolerance subjects (excluding the initial and final trials). It may be that the low tolerance subjects were maximizing the strategy of walking straight regardless of what the walk was like. However, the average curvature of both dose groups was similar, suggesting either that this was a measure subjects did not control or that it was affected by the speed or the directional properties of the walk. It may be a third type of measure-a characteristic of the "drunken walk" related to the general ataxic effects of alcohol.

MANOCOVA 2. Changes through time after ingestion are not readily evaluated when there are marked inter-subject differences in baseline response characteristics, without setting some initial and final values as covariates. It may be necessary to partial out both baseline and scale effects, which is equivalent to the affine transformation

$x^{\prime}=a+b x$

applied to all average total shifts in time on each measure, $x$, as a preliminary to examining differences within the time period of the experiment, between treatments. It was not worth utilizing all the 10 measures. After detailed examination of mean trends and the homogeneity of variance, only two measures,

$$
\begin{aligned}
& \mathrm{V} 1= \text { standard deviation of speed } \\
& \mathrm{V} 2= \text { average deviation about average } \\
& \text { speed }
\end{aligned}
$$

were retained. These are obviously related, but the form of their relation would depend on the shape of the distribution of recorded speeds over the path traversed. The measures were chosen because MANOVA 1 indicated that detection of $C D, C$, and $D$ effects and their changes during the time after ingestion would most probably be found economically within an analysis which used $\mathrm{C}$ and $\mathrm{D}$, and at each of 8 points in time (after 10, 20, 30. ., 80 minutes elapsed since drinking), the measures V1 and V2. A condensed notation V11 was used to indicate $V 1$ at 10 minutes, $\mathrm{V} t 1$ at $t$ minutes, $\mathrm{V} t 2$ for $\mathrm{V} 2$ at $t$ minutes. Here V11 and V12 have been used as covariates, and the remaining 14 observations have been used as dependent variables. The data represented by V91 and V92 were dropped to meet program capacity limitations.

The within cells regression, measuring the dependence of the variables on the covariates, gave one root with $F(25,2)=42.56, p<.023$. The regression coefficients of variables on covariates were as shown in Table 2 . As expected, the regression coefficients were all large and positive, which is appropriate for measures changing 
Figure 2

Average Speeds, Under Each of Four Conditions,

Over Time After Drinking

for Straight Line Walking Task

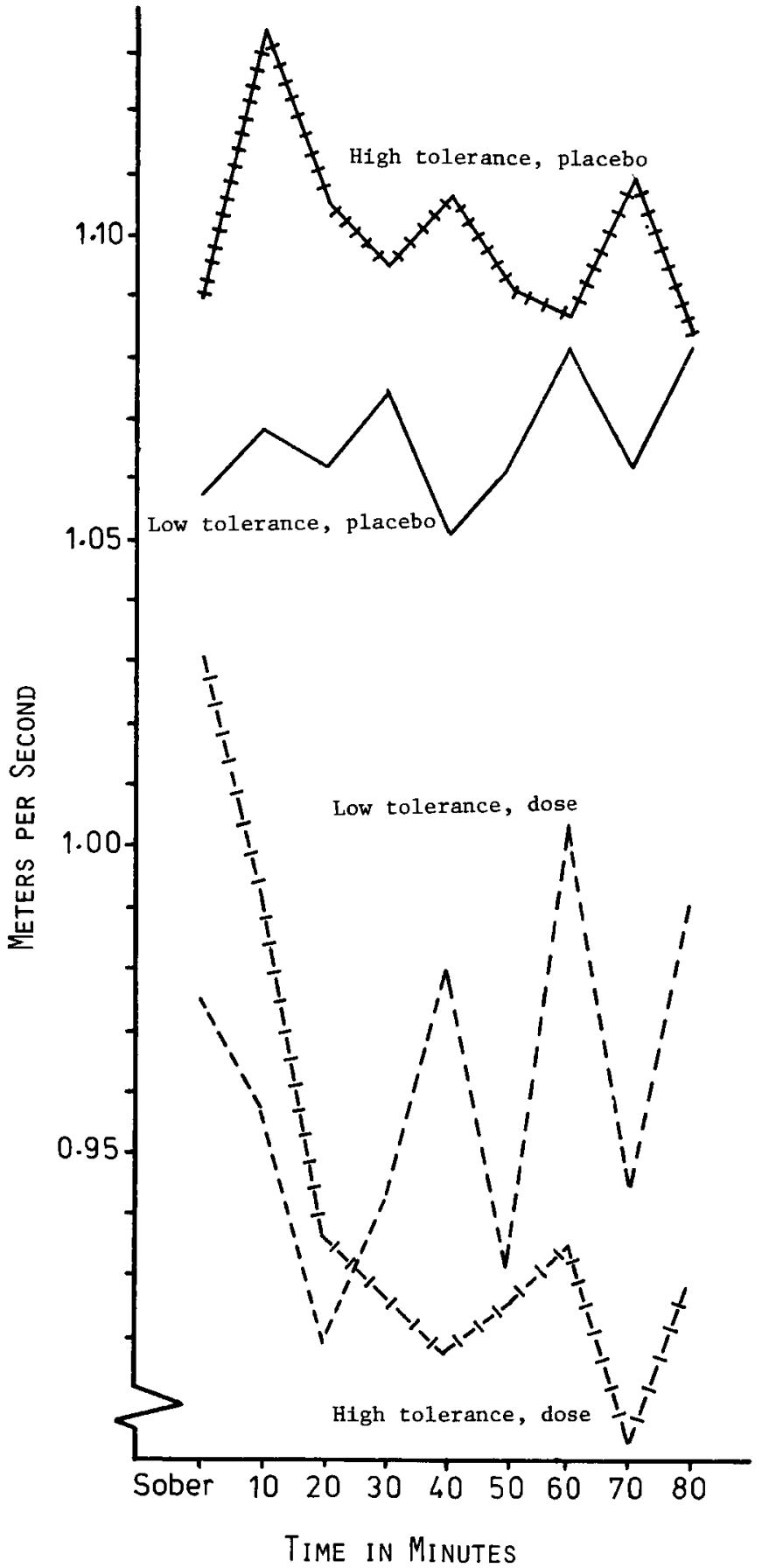


Figure 3

Standard Deviations of Average Speeds

Under Each of Four Conditions Over Time

After Drinking, for Straight Line Walking Task

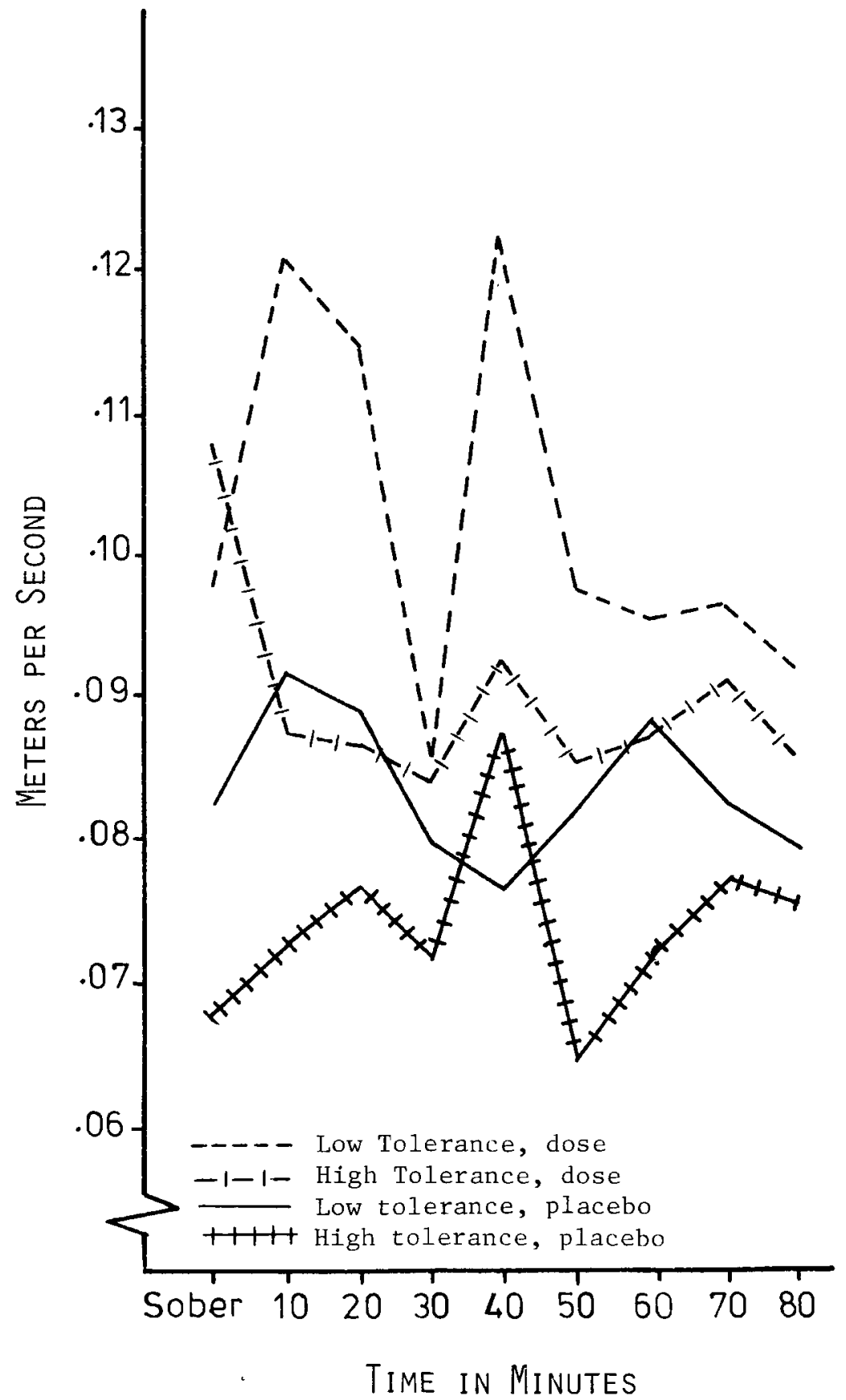


Table 2

Regression Coefficients with V11 and V12 as Covariates, for Straight Line Walking

\begin{tabular}{|c|c|c|c|}
\hline Variable & $\begin{array}{c}\text { Covariate } \\
\text { V11 } \\
\end{array}$ & Variable & $\begin{array}{c}\text { Covariate } \\
\text { V12 } \\
\end{array}$ \\
\hline V21 & .768 & V22 & .596 \\
\hline V31 & .709 & V32 & .579 \\
\hline V41 & .624 & V42 & .579 \\
\hline V51 & .601 & V52 & .621 \\
\hline V61 & .691 & V62 & .662 \\
\hline V71 & .754 & V72 & .611 \\
\hline V81 &.$\overline{642}$ & V82 & .740 \\
\hline
\end{tabular}

slowly over time. The return to the basal relative values of individual performance (up to a linear transformation) occurred after about 70 to 80 minutes; the points of return are underlined in Table 2.

In this multivariate analysis of covariance, there was no $C D$ interaction and no Dosage main effect after the baseline values were taken as covariates, but a $\mathrm{C}$ (tolerance) effect emerged $[F(14,1)=372.67, p<.041]$. This tolerance effect was almost entirely dependent on V32 (with V31 as a secondary contributing measure). That means large individual differences, related to prior tolerance for alcohol, showed up in performance deteriorations which did not correlate highly with initial differences in walking per- formance about 30 minutes after drinking and then subsequently disappeared.

MANOCOVA 3. The third analysis used four covariates, V11, V12, V81, and V82, and the remaining 12 measures as dependent variables. This analysis consequently partialed out some individual differences in linear trend with time over the duration of the experiment and left as the main source of dependent variation differences in the form of the changes in performance (that is, of the locus in time and the suddenness of the maximum deterioration and recovery therefrom).

The within cells regression of dependent variables on covariates again yielded one root $[F(48$, $5.9)=8.75, p<.006]$; and the regression coeffi-

Table 3

Regression Coefficients with Variables V11, V81, V12 and V82 as Covariates, for Straight Line Walking

\begin{tabular}{cccccc}
\hline \hline \multirow{2}{*}{ Variable } & \multicolumn{2}{c}{ Covariate } & & & \multicolumn{2}{c}{ Covariate } \\
\cline { 2 - 3 } \cline { 5 - 6 } V11 & V81 & Variable & V12 & V82 \\
V31 & .385 & .410 & V22 & .485 & .148 \\
V41 & .333 & .466 & V32 & .729 & .202 \\
V51 & .295 & .478 & V42 & .338 & .329 \\
V61 & .398 & .352 & V52 & .444 & .242 \\
V71 & .222 & .735 & V62 & .329 & .448 \\
& .213 & .773 & V72 & .554 & .078 \\
\hline
\end{tabular}


cients were as shown in Table 3 . It is seen by the data in Table 3 that V1 varied in a manner allowing a simple interpretation; the inter-individual differences decreased in their covariance with increasing separation in time, taken either from the beginning or the end of the sequence. There was no simple pattern for V2, although covariances with the baseline differences persisted. It could be that this measure shows deterioration later after drinking than does V1, but such a difference would not be readily interpretable.

Again, with the four covariates as with two, there were no $\mathrm{CD}$ or $\mathrm{D}$ effects. Instead, there was a $\mathrm{C}$ (tolerance) effect $[F(12,1)=358.85$, $p<.041$ ] with individual points in time contributing to a complex picture. V31, V32, V61, and $\mathrm{V} 71$ contributed, with the measures at $30 \mathrm{~min}$ utes dominant [univariate $(F(1,12)$ values for these $t$ measures were respectively, 6.0, 5.2, 4.2 and 4.1]. All other measures were negligible.

Overall, V1 is a more sensitive and better behaved indicator of individual differences in transient motor impairment as a function of pre-experimental tolerance. These differences appeared during the hour beginning 30 minutes after drinking for an ordinary unblindfolded walk.

\section{Complex Walk}

This task was devised for this study in order to create a difficult and tedious performance load which might maximize individual response differences under the different experimental conditions; it combined some of the features of a straight walk and the Romberg test. Therefore, precedents did not exist from which predictions might be made, although the Romberg test was used by previous investigators with simpler instrumentation (Begbie, 1966; Fregly et al., 1967).

The task of heel to toe walking is not so readily amenable to corrective strategies introduced by subjects who are partially aware of the degree of their own intoxication, because they do not usually walk in this very awkward fashion.
The same method of analysis was followed as for the straight walk, though here the measures which revealed the effects of treatment variables were different and more numerous; and their first-order intercorrelations changed. This task was susceptible in a wider sense to differences in individual deterioration associated with pre-experimental tolerance. In particular, the measures (\#5 and \#7) of RMS Dev. showed a high initial effect (at between 10 and 20 minutes after drinking) and a subsequent return to near baseline performance.

MANOVA 4. With no covariates removed, the overall effects $\mathrm{CD}, \mathrm{C}$, and $\mathrm{D}$ were again significant and there were no detectable effects over time, as shown in Table 4. In measuring CD interactions, variations in Av. Speed (or simple slowing up under intoxication) dominated other measures; this is shown in Table 4 by the contrasting values of the correlations between component variables and the canonical discriminant scores. The interaction of $\mathrm{C}$ and $\mathrm{D}$ was readily revealed in this slowing up to an extent which was not discernible in the straight walk, as shown in Figures 4 and 5.

For Dosage effects, 9 of the 10 available measures differentiated between placebo and dose. The remaining measure, Av. Dev. about St. Line (\#6) $[F(1,144)=4.68, p<.052]$, was borderline on the stringent criteria adopted here. The most sensitive measures of dosage effects were S.D. of Speed and Av. Dev. about Av. Speed. Note that S.D. of Speed correlated -.83 with the overall canonical discriminant score; average speed was the only measure which correlated positively with the overall canonical measure $(r=.210)$.

For C (tolerance) all measures except Av. Dev. about St. Line (\#6) differentiated. The exception was again borderline $[F(1,144)=3.5, p<.062]$; and the dominant measures were Acc. Av. Mag. and Av. Dev. about Av. Speed, which correlated respectively -.80 and -.76 with the overall canonical measure. Again, Average Speed was the only measure which correlated positively with the canonical discriminant score $(r=$ .361 ). 
Table 4

Results of the Multivariate Analysis of Variance Tests of Effects for the Complex Walk, Canonical Correlations $\left(\mathrm{R}_{\mathrm{c}}\right)$ and Correlations of Variables with Canonical Variates $\left(\mathrm{r}_{\mathrm{xv}}\right)$

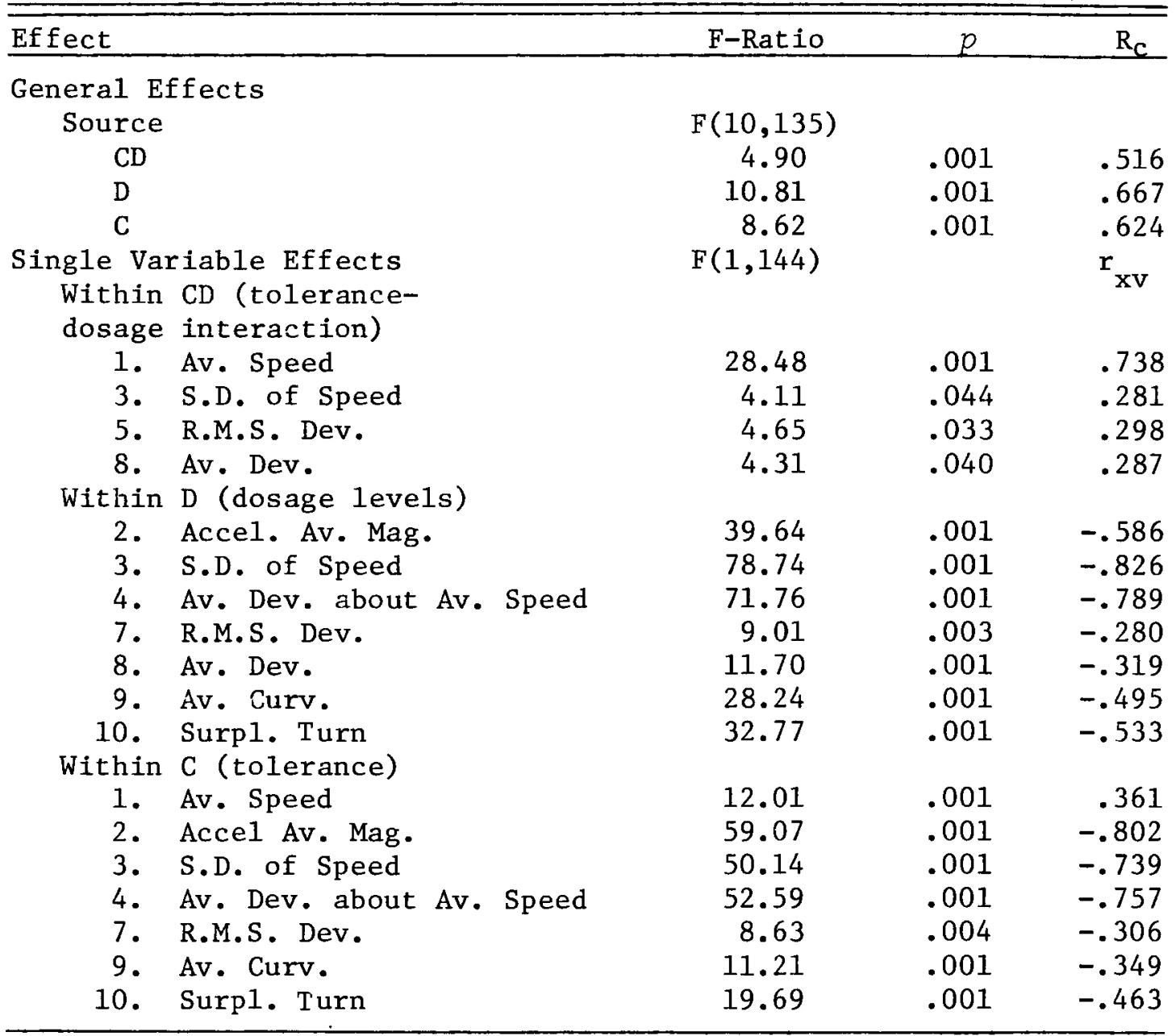

Note. Contrasts not shown were nonsignificant.

It is clear that in this complex walk, the factor structure of measures was different; the first order intercorrelations were high (ranging between -.304 and +.991) with Acc. Av. Mag., S.D. of Speed, and Av. Dev. about Av. Speed forming a tight cluster:

$\left|\begin{array}{lll}\mid .880 & & \mid \\ .886 & .989\end{array}\right|$

compared with

$\mid \begin{array}{lll}\mid .570 & & \mid \\ 1.088 & .227 & \mid\end{array}$

in the straight walk. Hence, a dynamic analysis of the two tasks would yield very different results; one walk certainly is not just a more difficult version of another. 
Figure 4

Average Speeds,

Under Each of Four Conditions,

Over Time After Drinking

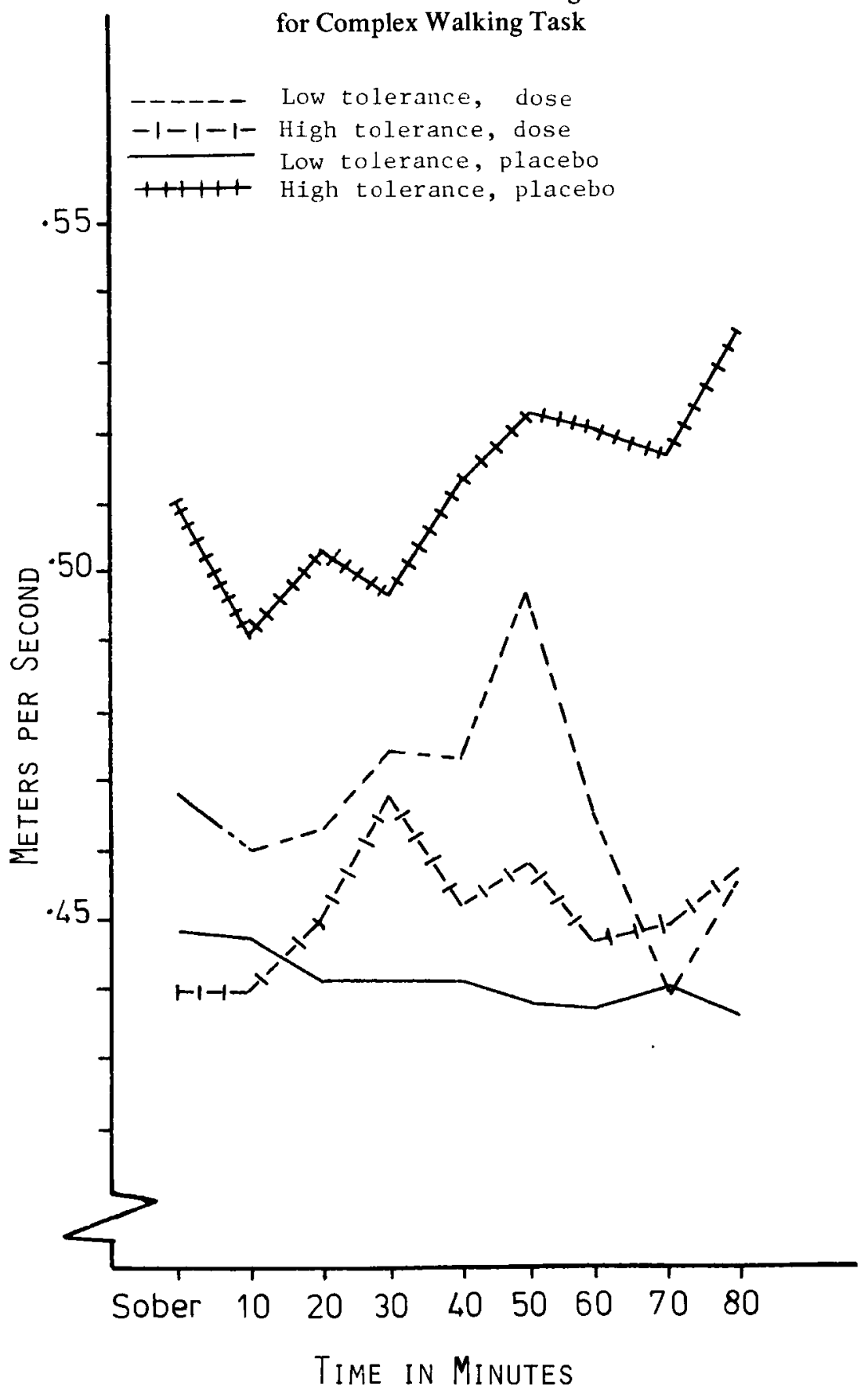




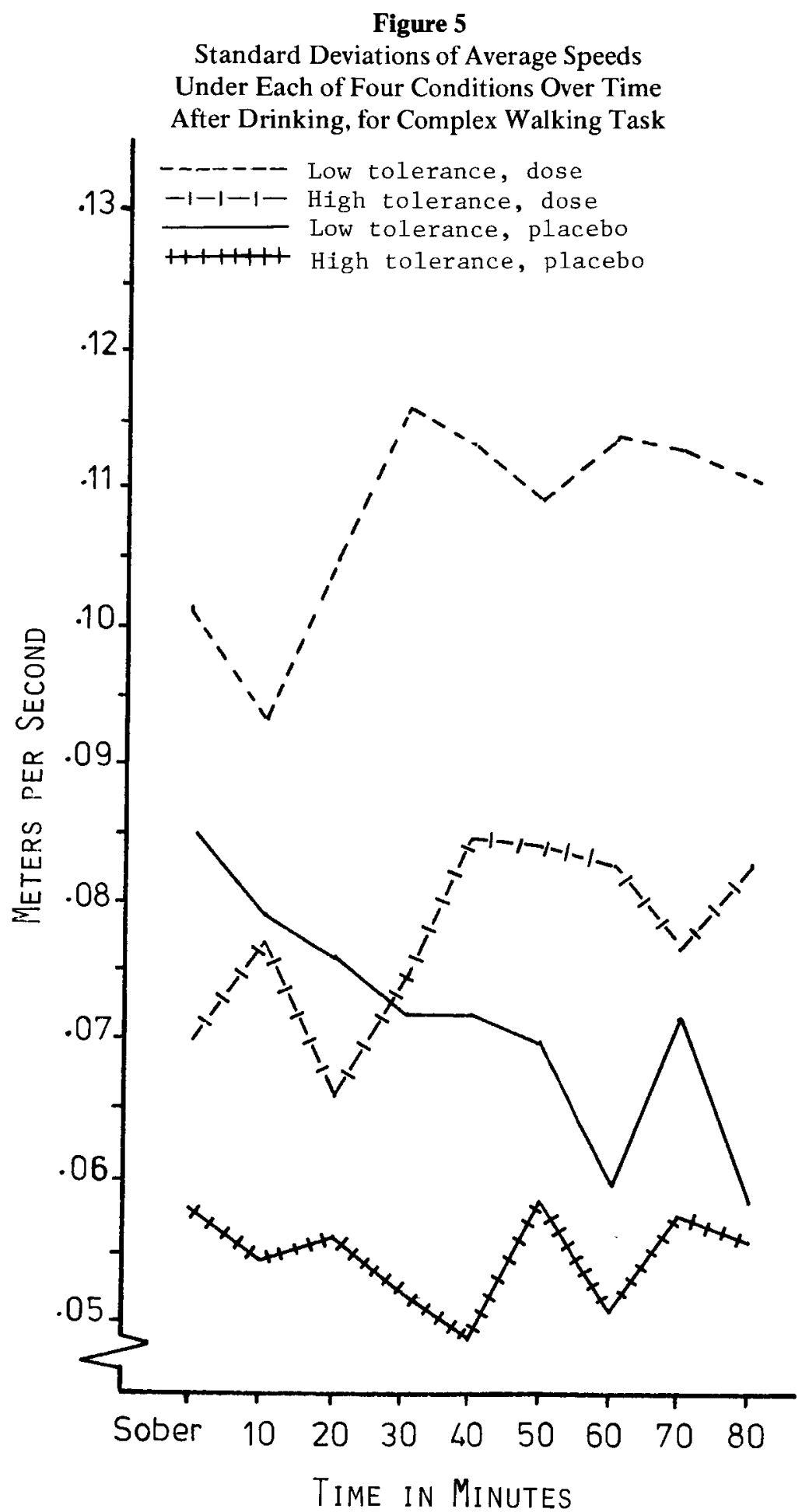


MANOCOVA 5. This corresponded exactly in structure with MANOCOVA 2; as before, $\mathrm{V} 11$ and $\mathrm{V} 12$ were the baseline covariates.

The within cells regression did not yield any significant roots, so the regression coefficients are not reported. None of the $C D, D$, or $C$ achieved significance; the most significant was D with $F(14,1)=3.31, p<.41)$. Thus, no contrasts were examined.

MANOCOVA 6. This corresponded exactly in structure with MANOCOVA 3; again, V11, $\mathrm{V} 12, \mathrm{~V} 81$, and V82 were the four covariates.

The within cells regression of variables on covariates yielded one significant root from the possible four $[F(48,5.9)=10.4, p<.004]$. The associated regression coefficients are shown in Table 5. There was little clear interpretable pattern in these data; marked individual differences in the rate and timing of changes in response generated the low covariances of temporally adjacent measures.

With four covariates removed, the Dosage factor emerged as significant $[F(12,1)=205.47$, $p<.054]$. This was the only multivariate component which did so; $C D$ and $C$ had probabilities of .252 and .534 , respectively.

The different effects of dosage showed strongly at V51, V52, V71, and V72; all other measures were of negligible importance. [The $F(1,12)$ univariate values for the four measures were respectively $4.25,4.06,10.86$, and 4.94 ].
The interesting difference, then, is that the complex walk is only apparently sensitive to Dosage differences but not to Tolerance differences, once the baseline levels and linear drift with time are partialled out as covariates. This contrasts with the Straight walk, which was only susceptible to Tolerance effects and not to Dosage.

It is clear that the short-term effects of alcohol on motor skills vary considerably as a function of the familiarity of the task, and the probability of detecting such effects can vary markedly with the measureschosen. Hence, generalizations about the effects of acquired tolerance for alcohol with prolonged drinking experience would be very difficult to justify, but could be misleadingly supported by a narrow selection of test procedures.

\section{Conclusions}

Before attempting to draw implications from these results, it is expedient to qualify what is meant by "tolerance." The "high tolerance" subjects used here were not chronic alcoholics with years of heavy drinking experience, but simply the high consumption end of an unsystematic sample of university students. There may be some diagnosable alcoholics in the academic population, but they are not demonstrably present in this study. The tolerance for high

Table 5

Regression Coefficients with Variables V11, V81, V12 and V82 as Covariates, for Complex Walk

\begin{tabular}{lllllr}
\hline & \multicolumn{2}{c}{ Covariate } & & \multicolumn{2}{c}{ Covariate } \\
\cline { 5 - 6 } Variable & V11 & V81 & Variable & V12 & V82 \\
\hline V21 & .626 & .266 & V22 & .146 & -.039 \\
V31 & .448 & .636 & V32 & .009 & -.402 \\
V41 & .063 & .924 & V42 & .002 & .304 \\
V51 & .195 & .451 & V52 & -.089 & .265 \\
V61 & .005 & .786 & V62 & -.161 & -.389 \\
V71 & .511 & .117 & V72 & .221 & .052 \\
\hline
\end{tabular}


blood alcohol levels is lower in inexperienced and younger drinkers, so predictions concerning the performance of heavy drinkers in the 30-50 year age groups cannot be made.

The effects of dosage are generally confounded with those of tolerance, but detectable performance impairment will vary in form and extent with task familiarity and complexity. Again, these latter two variables must also be confounded in any studies of drug actions upon the performance of complex skills.

The more experienced drinker may initially (for 30-40 minutes) show skill in compensation for the deleterious actions of alcohol. After a further period (up to 70 minutes) the differences in performance between high and low tolerance subjects tend to diminish into insignificance. How much this is due to fatigue and how much to progressive detoxification-estimated by Watson and Batt (1976) to occur at an equivalent rate of $17.5 \mathrm{mg} / 100 \mathrm{ml} /$ hour, so that the intoxicated subjects would be fully sober again in about 5 hours-has not been estimated.

For the complex walk in which no practical corrective strategy is available to the high tolerance subject, although there was a sudden and marked decrement in performance, recovery occurred again to a satisfactory level. The low tolerance subjects also showed this initial drop and recovery in all tasks examined, so that the difference between tolerance levels disappeared in the complex and unfamiliar walk, but the difference between dosage levels was accentuated.

The practical implications are obvious: A diversity of tasks and measures are needed to probe the subtle dynamics of alcohol actions on the higher nervous system with its complex changes over time. A lack of validity of simple tasks performed at one point in time in predicting impairment of motor skills, such as driving a car, could easily arise when subjects are drawn from populations varying greatly in time elapsed since drinking, dosage, and tolerance.

\section{References}

Begbie, G. H. The effects of alcohol and of various amounts of visual information on a balance test. Ergonomics. 1966, 9. 325-333.

Bowman, R. S., Stein, L. I., \& Newton, J. R. Measurement and interpretation of drinking behavior. Journal of Studies on Alcohol, 1975, 36. 1154-1172.

Carlson, A. J., Kleitman, N., Muehlberger, C. W., McLean, F. C., Gullicksen, H., \& Carlson, R. B. Studies on the possible intoxicating action of $3.2 \%$ beer. Chicago: University of Chicago Press, 1934.

Fregly, A. R., Bergsteadt, M., \& Graybiel, A. Relationship between blood alcohol, positional alcohol nystagmus, and postural equilibrium. Quarterly Journal of Studies on Alcohol, 1967, 28, 11-21.

Goldberg, L. Quantitative studies of alcohol tolerance in man. The influence of ethyl alcohol on sensory motor and psychological functions referred to blood alcohol in normal and habituated individuals. Acta Physiologica Scandinavica. 1943, 5, Supplement 16, 1-128.

Hebbelinck, M. The effects of a small dose of ethyl alcohol on certain basic components of human physical performance. II The effects of neuromuscular performances. Archives of Internal Pharmacodynamics, 1963, 143, 247-257.

Ideström, C. M., \& Cadenius, B. Time relations of the effects of alcohol compared to placebo. Psychopharmacologia, 1963, 13, 189-200.

Kay, L. Towards objective mobility evaluation. Some thoughts on a theory. New York: American Foundation for the Blind, 1974.

Laves, W. Mass und Zahl in der medizinschen Begutachtung der Fahrt üchigleeit. Medizinische Klinik, 1955, 50, 9-12.

Miles, W. R. Static equilibrium. Methods in Medical Research. 1950, 3, 157-65.

Pihkamen, T. A. Neurological and physiological studies on distilled and brewed beverages. Helsinki, Maalaisleintien Liiton Korjapaino, 1957. also Annales medicinae experimentalis et biologiae Fenniae, 1957, 35. Supplement 9, 1-152.

Powell, B. J., Goodwin, D. W., \& Bremer, D. Drinking experience versus personality factors as predictors of tolerance for alcohol. British Journal of Psychiatry, 1973, 122, 415-417.

Reid, L. D.. \& Ibrahim, M. F. K. The application of human operator describing functions to studies on the effects of alcohol and marijuana on human performance. IEEE Transactions on Systems, Man and Cybernetics, 1975, SMC-5, 506-519.

Schmidt, $O$. Die elderische registrierung akloholbedingter gleichgewichtsstörungen (Electrical record- 
ing of disturbances of equilibrium caused by alcohol). Deutsche Zeitschrift für die Gesamte Gerichtliche Medizin, 1956, 45, 231-235.

Sheridan, T. B., \& Ferrell, W. R. Man-machine systems. Cambridge, MA: M.I.T. Press, 1974.

Strelow, E. R., Brabyn, J. A., \& Clark, G.R.S. Apparatus for measuring and recording path velocity and direction characteristics of human locomotion. Behaviour Research Methods and Instrumentation. 1976, 8, 442-446.

Vogel, M. Low blood alcohol concentrations and psychomotor performance. An exploratory study. Quarterly Journal of Studies on Alcohol, 1958, 19. 573-589.

Watson, P. E., \& Batt, R. D. Quantities of alcohol consumed likely to result in blood alcohol levels exceeding $100 \mathrm{mg} / 100 \mathrm{ml}$ blood. Palmerston North, New Zealand: Massey University, 1976.

\section{Acknowledgements}

The vodka used in this research was donated by Fletcher Humphreys and Co., Christchurch. New Zealand.

\section{Author's Address}

R.A.M. Gregson, Department of Psychology, University of Canterbury, Christchurch 1, New Zeland. 